\title{
Jitter Measurement in Digital Signals by Using Software Defined Radio Technology
}

\author{
Gehan Anthonys*, Michael J. Cree ${ }^{\dagger}$ and Lee Streeter ${ }^{\ddagger}$ \\ School of Engineering, University of Waikato, Hamilton 3240, New Zealand \\ Email: *gehan.anthonys@gmail.com, $\left\{{ }^{\dagger}\right.$ michael.cree, ${ }^{\ddagger}$ lee.streeter $\} @$ waikato.ac.nz
}

\begin{abstract}
Here, we propose a method using software defined radio (SDR) technology to measure periodic and random jitter in a digital signal. Conventional methods to measure jitter required expensive measurement equipment. We use a cheap (less than US \$40) SDR USB dongle to expose random and periodic jitter in the amplitude modulated light source of a range camera. By using Fourier analysis we generate an ideal reference signal to enable to extract jitter at the zero crossings of the signal under test. We measure jitter with SDR receiver and more conventionally a real-time digital oscilloscope. From the SDR receiver, we find the periodic jitter at low frequency and compare to the oscilloscope results. We demonstrate that periodic and random jitter can be detected on a RF signal with consumer priced products provided that the jitter frequency less within the bandwidth of the receiver.
\end{abstract}

Index Terms-jitter measurement, software defined radio, Fourier analysis, down-mixing

\section{INTRODUCTION}

Digital signals are never perfect in real world applications. Generally, a digital signal can deviate from the ideal signal in amplitude and in displacement in time. If the deviation is in amplitude, it is referred to as amplitude noise (or simply noise) while a deviation in time is referred to as jitter [1]. The jitter in a signal can be measured by time domain or frequency domain methods. Often we can measure jitter in a signal by using instruments such as a real-time digital oscilloscope, pulse/pattern generator or spectrum/logic analyser by graphical representation methods such as the eye pattern, histogram, bathtub plot [2]. These methods require an accurate reference clock to make the jitter measurement.

A general purpose automated search and non-linear fitting algorithm for jitter separation method in the time-domain was introduced by Li et al. [3]. They fitted the tail parts of the jitter histogram with non-linear jitter models and calculated a bit error rate for each category of jitter. Dou et al. [4] used the time lag correlation (TLC) functions for each type of jitter and presented a set of equations for jitter calculation. In order to reduce the calculation time, Pang et al. [5] used the fast Fourier transform (FFT) in addition to the TLC which reduces the number of equations to solve. All of the above methods require a reference clock. Random and periodic jitter were extracted without a reference clock in simulated noisy signals by calculating the energy of the jitter spectrum by Anthonys et al. [6]. They used Fourier analysis to construct the ideal signal from the signal with jitter. In all the above cases expensive medium frequency $(>\mathrm{GHz})$ measurement equipment is required.

In the late twentieth century, a new technology fusing software and digital radio called software defined radio (SDR) became the choice for wireless communication. SDR is a type of reconfigurable radio in which some or all of the physical layers of functionality are implemented in software and/or firmware [7]. SDR consists of an antenna and an analogue-digital converter (ADC) on the receiver side and a digital-analogue converter (DAC) and an antenna on the transmitter side. The rest of the functionality is handled by reprogrammable processors [8]. The hardware components of SDR are available at affordable prices and software implementations are freely available. At present, the price of a SDR receiver can be less than US $\$ 40$ [9]. The SDR can be totally configured by the software so that a common platform can be used in many number of areas [10]-[14]. In SDR technology, the signal from the antenna is directly converted to the digital domain and all the further processing is under software control.

In this paper, we explore using an inexpensive SDR receiver connected to a computer, coupled with our previous investigated method based on signal processing [15] to measure the jitter in a digital signal. We use the emitted light source from a time-of-flight ( $\mathrm{ToF}$ ) camera with known jitter as the signal with jitter to be tested. We compare the jitter with the signal acquired with the SDR to the same signal acquired by a medium speed oscilloscope and show that periodic jitter at low frequency can be measured with the inexpensive USB SDR receiver.

The rest of the paper is structured as follows. We give a brief introduction to SDR and the jitter measurement technique in Section II. The proposed method with experimental set up is described in Section III, which is followed by results and discussion.

\section{BACKGROUND}

We give a brief review of jitter in signals then an overview of the relevant operational principle of SDR technology in this section.

\section{A. Jitter in Signals}

Fig. 1 shows that the deviation of the signal $n$th timing event $t_{n}$, from its reference (ideal) occurrence in time axis only, is given by

$$
\Delta t_{n}=T_{n}+\Delta t_{n-1}-T,
$$


where $T$ is the period of the ideal signal and $T_{n}$ is the $n$th period (cycle) of the signal given by $T_{n}=t_{n+1}-t_{n}$.

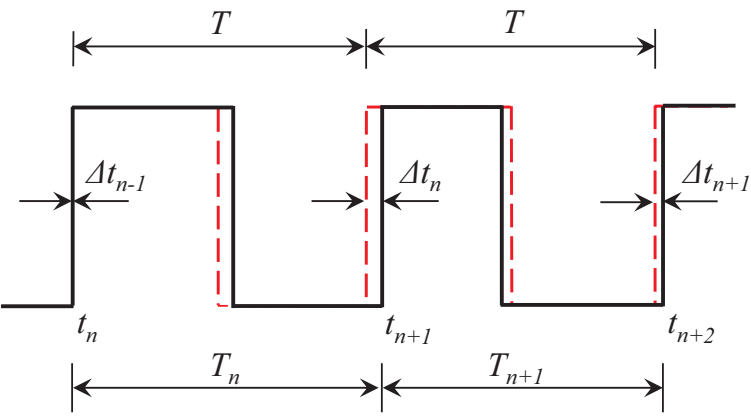

Ideal signal $---\quad$ Jittery signal -

Fig. 1. Jitter representation in a signal.

Jitter can be categorized as non-deterministic or deterministic. Non-deterministic jitter is jitter due to random process; and is commonly referred to as random jitter (RJ). RJ often follows a Gaussian distribution, thus can be characterized by its root-mean-square (RMS) value. Deterministic jitter (DJ) is any jitter that is deterministic in nature and is linearly additive. DJ can be further categorized as periodic jitter (PJ), bounded correlation jitter and various data dependent jitters [1]. RJ in a signal is caused by various random processes such as white noise, thermal noise, shot (Poisson) noise, flicker noise [16]. DJ is caused by such things as signal modulation, crosstalk and reflections [17].

\section{B. Software Defined Radio Technology}

SDR consists of a transmitter and a receiver. It is the receiver that we are primarily interested in. In a SDR receiver, the signal from the antenna passes through wideband low-noise amplification, down-conversion to a lower frequency, filtering before distribution. The basic structure of the SDR receiver is shown in Fig. 2.

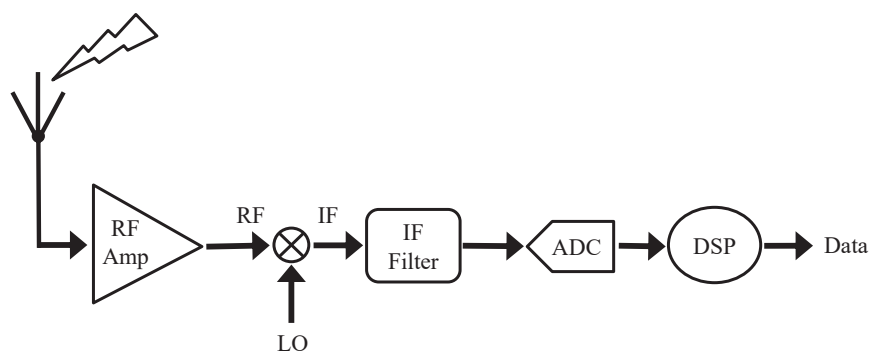

Fig. 2. Basic structure of a SDR receiver.

We use a RTL-SDR USB receiver that consists of the Rafael R820T2 tuner (the RF amp through to IF filter stages) coupled to a RTL2832U demodulator (the ADC/DSP stages). The signal enters via the antenna and is amplified by a radiofrequency (RF) tuner then is downshifted by mixing with the local oscillator (LO) frequency to the intermediate-frequency
(IF). The IF signal is bandlimited (IF filter) before being sampled by the ADC at a fixed rate of $28.8 \mathrm{MHz}$, then passed through a digital downconverter, a lowpass-filter and into a resampler (which then downsamples to the desired sample rate, 3.2 $\mathrm{MHz}$ at maximum). Therefore, from this SDR receiver we can only measure frequencies below 1.6 MHz after down conversion.

We wish to extract jitter in the amplitude modulate light source of a range imaging camera. The ToF cameras are based on the phase difference $\phi$ between the emission of the signal and its return to shuttered pixelated sensor, after being reflected by an scene and it is believed that jitter can affect the phase measurement.

\section{Methodology}

Here, we propose a general method to calculate the jitter in a digital signal captured with SDR technology or by oscilloscope. We follow with a description of the experimental setup to measure the jitter in the emitted light signal of a depth camera by using the SDR USB dongle.

\section{A. The proposed approach}

The main steps of the methodology used to measure the jitter parameters in a digital signal as a flow chart, is shown in Fig. 3.

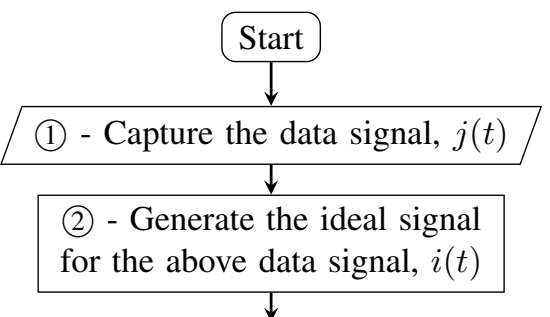

(3) - Align the signals $j(t)$ and $i(t)$ with respect to the first zero crossing points of either rising or falling edges $\downarrow$

(4) - Obtain the exact zero crossing points at aligned edges for each signal $\downarrow$

(5) - Calculate the jitter amount (time deviation) for each cycle $\downarrow$

(6) - Obtain the frequency spectrum of the time deviations obtained in (5)

(7) - Calculate the jitter parameters from the spectrum

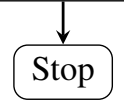

Fig. 3. Flow chart of the jitter measurement in a digital signal.

We capture the light (data) signal of the camera as our first step. In order to clearly identify the zero crossings of the signal, we smooth it with minimal impact on the signal by using the Savitzky-Golay filtering algorithm [18]. Then, 
we generate the corresponding reference (ideal) signal for the camera as step (2). The maximum amplitude of the smoothed data signal and its corresponding exact frequency (by performing quadratic interpolation) of Fourier spectrum are used as the amplitude and frequency, respectively, for generating the ideal signal. Further more, we repeat this analysis for many data sets and then take the mean frequency among them as for the exact frequency of the ideal signal for the camera. For step (3), the data and ideal signals are aligned with respect to the first zero crossing point of either rising or falling edges. Next, the zero crossing points at aligned edges are estimated for each signal by using linear interpolation, as for step (4).

The jitter $\Delta t$ at the aligned edge of each cycle is computed by taking the time difference between the zero crossing of data and ideal signals of the camera as for step (5). The length of the signal is somewhat arbitrary, thus it is not normal to obtain a complete number of cycles of the base frequency of the signal. To reduce the impact of spectral leakage, we take the floor of the number of cycles in the jitter signal as

$$
N_{\Delta t}=\text { floor }\left(\frac{f N_{s}}{F_{s}}\right),
$$

where $f$ is the signal frequency, $N_{s}$ is the number of samples in the acquired signal, and $F_{s}$ is the sample rate of the acquisition.

It is an assumption of the $N$-point DFT that the signal is repeated every $\mathrm{N}$-points throughout all time, however, the frequency of any present periodic jitter may not divide $N$, this not repeat at complete cycles, resulting in a discontinuity of the signal, leading to spectral leakage. By using a windowing function, the effects of the spectral leakage is minimized, but this leads to a decrease in the total energy of the signal. Therefore an energy correction factor must be used. We use the Hanning window [19] and its energy correction factor is given by [20]

$$
\mathrm{E}_{H}^{c f}=\left[\int_{0}^{1}\left(\sin ^{2}\left(\frac{\pi n}{N-1}\right)\right)^{2} \mathrm{~d} t\right]^{-\frac{1}{2}}=\sqrt{\frac{8}{3}},
$$

where $n=0,1, \ldots, N-1$. Then, to compute step (6), we obtain the frequency spectrum of the jitter signal by DFT. Finally, the corresponding jitter parameters for the camera are calculated by the energy of the spectrum [15]. As an example, the total energy in the spectrum is obtained by [21]

$$
\mathrm{E}(\Delta t)=\mathrm{E}_{H}^{c f} \sum_{k=0}^{N-1}\left|\mathscr{F}_{k}\right|^{2},
$$

where $\mathscr{F}_{k}$ is the DFT of the $k$ th sample of the jitter signal. Then, the amplitude of the total jitter of the signal is

$$
\Delta t=\sqrt{2 \mathrm{E}(\Delta t)},
$$

where the factor $\sqrt{2}$ is the conversion of the RMS of a sinusoid to its amplitude. In the case of both RJ and PJ present, one has to find the PJ first by DFT [15]. Note that, we compensated the results of the SDR setup by the ratio of the number of samples per cycle corresponding to the SDR receiver and oscilloscope.

\section{B. Experimental setup}

We captured the emitted light signal of a MESA SR4000 range imaging camera by using a silicon biased photodetector DET025A. We used two different devices for the measurements, a SDR USB dongle (RTL2832U) and a real-time digital oscilloscope (HP Infiniium 54846B), separately. The range imaging camera was set to produce a $30 \mathrm{MHz}$ modulated signal which we had characterised previously [15]. For the control experiment we generated $30 \mathrm{MHz}$ sine waves with a signal generator (HP Agilent 8648B). Both signal sources were analyzed by the SDR USB dongle and by oscilloscope. We used the same coaxial cable for all experiments. The experimental setup with the components used is shown in Fig. 4.

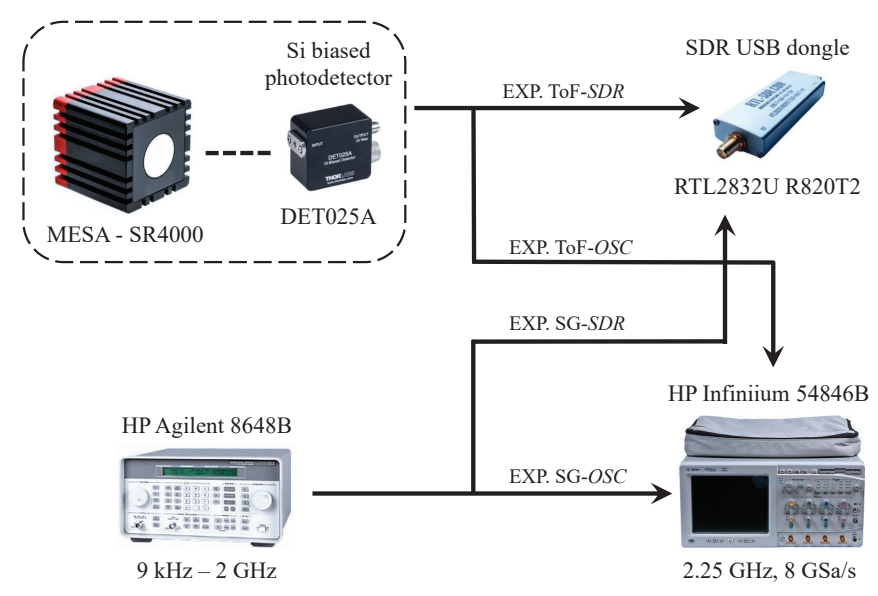

Fig. 4. Experimental components and setup.

For the SDR setup, we down mixed the emitted light signal (30 MHz) of the camera with $29.6 \mathrm{MHz}$ as the RF tuner LO frequency to give a $400 \mathrm{kHz}$ IF signal at the ADC. For the SDR, the parameters are $f=400 \mathrm{kHz}, N_{s} \approx 10960$ and $F_{s}$ $=3.2 \mathrm{MSa} / \mathrm{s}$. For oscilloscope setup, these are $f=30 \mathrm{MHz}$, $N_{s}=2^{16}$ and $F_{s}=8 \mathrm{GSa} / \mathrm{s}$. These are tabulated in Table I. We obtained the number of jitter samples $N_{\Delta t}$ by using (2) for each measurement setup as tabulated in column three of Table I. The jitter spectrum for each acquisition is obtained by using Steps (1) to (6) in Fig. 3. In order to reduce the uncertainty of the result, we captured 1000 acquisitions for each experiment with the oscilloscope and 8000 acquisitions for each experiment with the SDR setup. However, about 3\% of the acquisitions with the SDR were found to contain errors and were discarded from analysis. It appears that the SDR (or its device driver) occasionally drops samples [22].

\section{EXPERIMENTAL RESUlTS AND DISCUSSION}

Fig. 5 shows the corresponding result for step (3) for the single acquisition of SR4000. The data and ideal signals are aligned with respect to the first zero crossing points. Table II tabulates the experimental parameters for each setup. The fourth column represents the number of samples per cycle for each setup. The last column is the corresponding frequency 
TABLE I

PARAMETERS OF THE COMPONENTS AND SETUP.

\begin{tabular}{|c|c|c|c|}
\hline Signal source & $\begin{array}{l}\text { Measuring } \\
\text { equipment }\end{array}$ & $\begin{array}{c}\text { No. of cycles } \\
N_{\Delta t} \text { by (2) }\end{array}$ & EXP. \\
\hline MESA SR4000 & \multirow{2}{*}{$\begin{array}{c}\text { SDR USB dongle } \\
3.2 \mathrm{MSa} / \mathrm{s} \\
\text { (RTL2832U) }\end{array}$} & \multirow[b]{2}{*}{1344} & ToF-SDR \\
\hline $\begin{array}{l}30 \mathrm{MHz} \text { signal } \\
\text { (Agilent } 8648 \mathrm{~B} \text { ) }\end{array}$ & & & SG-SDR \\
\hline MESA SR4000 & \multirow{2}{*}{$\begin{array}{c}\text { Oscilloscope } \\
2.25 \mathrm{GHz}, 8 \mathrm{GSa} / \mathrm{s} \\
\text { (Infiniium-54846B) }\end{array}$} & \multirow[b]{2}{*}{245} & ToF-OSC \\
\hline $\begin{array}{l}30 \mathrm{MHz} \text { signal } \\
\text { (Agilent } 8648 \mathrm{~B} \text { ) }\end{array}$ & & & SG-OSC \\
\hline
\end{tabular}

with the standard deviation (STD) of the ideal signal which is extracted from the data, for each experimental set up. Each of these STDs are based on a large number of data sets (i.e., 1000 for OSC setup and $\approx 8000$ for SDR setup). The frequencies of the signals are listed in Table II and are determined very accurately. This is used to generate the ideal signal.

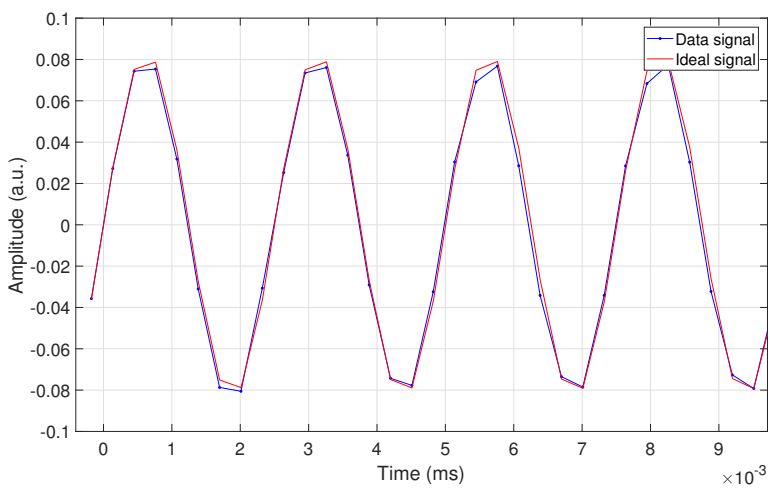

(a)

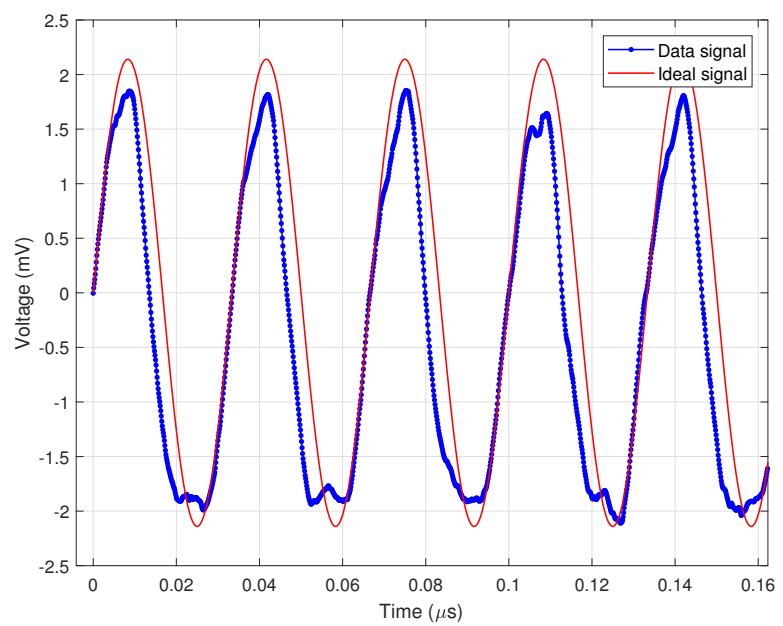

(b)

Fig. 5. Aligned the light source (data) signal and ideal signal for a single acquisition of SR4000 by using the setup: (a) SDR and (b) Oscilloscope.

After step (6), we obtained jitter spectra for MESA SR4000 and $30 \mathrm{MHz}$ signal for each experimental setup, separately, as

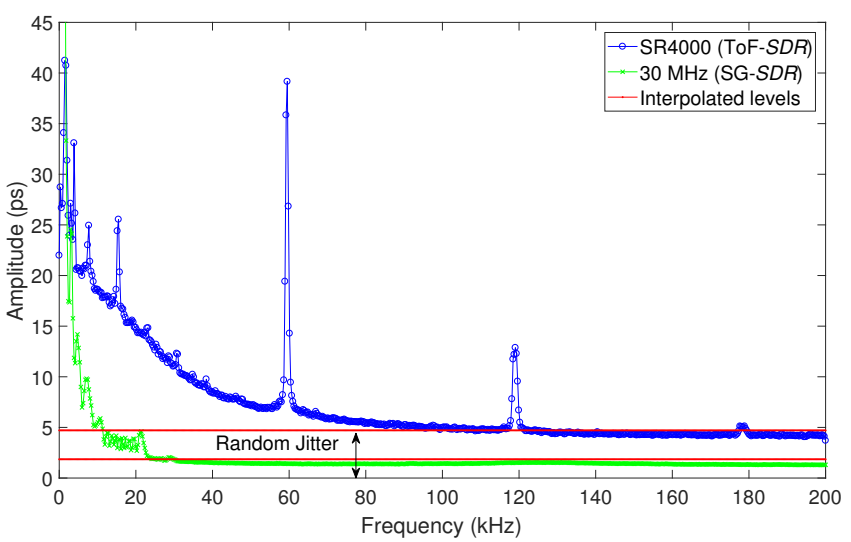

(a)

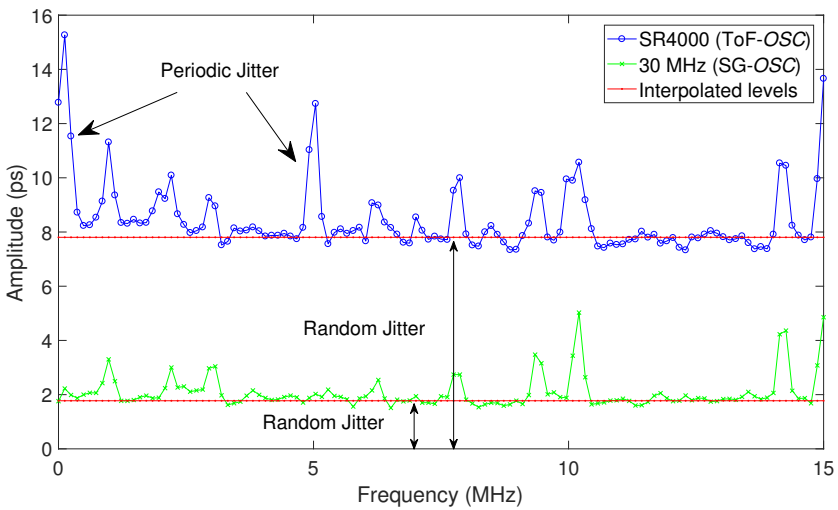

(b)

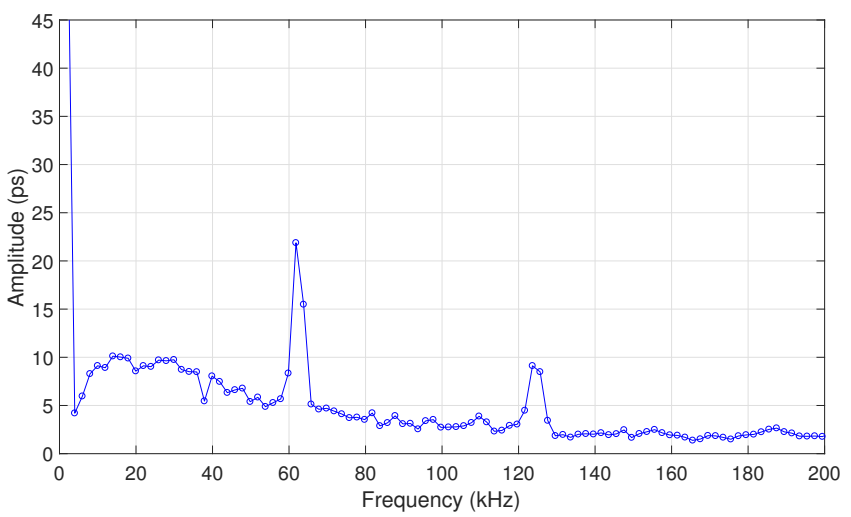

(c)

Fig. 6. Jitter components for the SR 4000 and $30 \mathrm{MHz}$ signal by the setup: (a) SDR receiver (b) Oscilloscope and (c) an expansion version of first 200 $\mathrm{kHz}$ of the result acquired with a faster oscilloscope (Agilent DSOS 604A).

shown in Fig. 6. The jitter components and the interpolated level between periodic and random jitter for each signal is marked in the figure. The frequency range for the SDR setup and oscilloscope setups are $0-200 \mathrm{kHz}$ and $0-15 \mathrm{MHz}$, respectively. Fig. 6(c) is an expansion version of the first $200 \mathrm{kHz}$ of the oscilloscope result acquired with a faster 
TABLE II

EXPERIMENTAL PARAMETERS

\begin{tabular}{|c|c|c|c|c|}
\hline \multicolumn{2}{|c|}{ EXP. setup } & Sampling & No. of & Ideal signal's \\
\hline ToF & \multirow{2}{*}{$\begin{array}{c}S D R \\
(400 \mathrm{kHz})\end{array}$} & \multirow{2}{*}{$312.5 \mathrm{~ns}$} & \multirow{2}{*}{8} & $399.614 \pm 0.0017 \mathrm{kHz}$ \\
\hline SG & & & & $399.779 \pm 0.0001 \mathrm{kHz}$ \\
\hline ToF & \multirow{2}{*}{$\begin{array}{c}\text { OSC } \\
(30 \mathrm{MHz})\end{array}$} & \multirow{2}{*}{$125 \mathrm{ps}$} & \multirow{2}{*}{$\frac{800}{3}$} & $30.006 \pm 0.0012 \mathrm{MHz}$ \\
\hline SG & & & & $30.006 \pm 0.0006 \mathrm{MHz}$ \\
\hline
\end{tabular}

oscilloscope - Agilent DSOS 604A (6 GHz, $20 \mathrm{GSa} / \mathrm{s}$ ).

Note that, we compensated the results from SDR setup by the ratio 1:100/3 (see fourth column of Table II). From Fig. 6(b) (from the oscilloscope measurements), it is straightforward to identify two high peaks at 0.12 and $5.04 \mathrm{MHz}$ with the amplitudes 61 and $34 \mathrm{ps,} \mathrm{respectively,} \mathrm{on} \mathrm{the} \mathrm{jitter} \mathrm{signal} \mathrm{of}$ SR4000 (i.e., ToF-OSC) that are not present in the control 30 $\mathrm{MHz}$ signal, thus with the oscilloscope it is quite clear which peaks are $\mathrm{PJ}$ from the device under test. There are no periodic jitter in $30 \mathrm{MHz}$ signal from signal generator for both cases (see Fig. 6(a) and (b)). We found the PJ at $59.52 \mathrm{kHz}$ with the amplitude of $164 \mathrm{ps}$ from the SDR setup. This can verify by the result from the fast oscilloscope shown in Fig. 6(c) which better existing the frequency components below $120 \mathrm{kHz}$ than that shown in Fig. 6(b).

There is a broad and flat spectrum being the RJ in the camera. We calculate the jitter parameters by considering the full spectrum for each experiment as tabulated in Table III. Make clear that the PJ at $59.52 \mathrm{kHz}$ is represented both

TABLE III

EXTRACTED RESUlTS FROM MEASUREMENTS

\begin{tabular}{|c|c|c|c|}
\hline \multicolumn{2}{|c|}{ EXP. setup } & $\begin{array}{c}\text { Periodic jitter } \\
{\left[f_{P J}^{(p)}, A_{P J}^{(p)}(p s)\right]}\end{array}$ & $\begin{array}{c}\text { Random jitter } \\
\sigma_{R J}(\mathrm{ps})\end{array}$ \\
\hline \multirow{2}{*}{$\approx$} & ToF & {$[59.52 \mathrm{kHz}, 164]$} & 220 \\
\cline { 2 - 4 } & SG & - & 87 \\
\hline \multirow{2}{*}{} & ToF & {$[0.12 \mathrm{MHz}, 61]$ and $[5.04 \mathrm{MHz}, 34]$} & 160 \\
\cline { 2 - 4 } & SG & - & 35 \\
\hline
\end{tabular}

by SDR receiver and the faster oscilloscope (Agilent DSOS 604A). But from the medium speed oscilloscope (Hp 54846B), we are unable to find this frequency. The random jitter of 220 and $160 \mathrm{ps}$ are obtained from SDR setup and oscilloscope, respectively. The measurements of the random jitter in signal generator from the SDR and oscilloscope setups are found as 87 and $35 \mathrm{ps}$, respectively. We believe the oscilloscope results are well accurate.

\section{CONCLUSION}

The main aim of this study is to use the SDR technology to measure the jitter in digital signals. We experimented with a cheap SDR USB dongle with previously investigated method to measure the jitter on the emitted light signal of MESA SR4000 a depth camera. One advantage of this analysis is, the methodology we used does not required a reference (ideal) clock to trigger the time measurement unit. We obtained the frequency of the reference signal from its data by using the Fourier analysis and used the maximum amplitude among that data as the corresponding amplitude for reference signal. We downshifted the signal to a low frequency and as a result of that we were able to find the periodic jitter at lower frequencies (less than 1.6 MHz). By performing the energy calculation of the jitter spectrum we calculated the relevant jitter parameters for the camera. We believe, with the high sample rates and high quality of SDRs can be used to explore periodic jitter at least at lower frequencies in a RF signal.

\section{REFERENCES}

[1] M. Li, Jitter, Noise, and Signal Integrity at High-speed, 1st ed. Upper Saddle River, NJ, USA: Prentice Hall, 2007.

[2] J. Hancock et al., "Jitter-understanding it, measuring it, eliminating it part 1: Jitter fundamentals," High Frequency Electronics, vol. 4, no. 4, pp. 44-50, 2004.

[3] M. P. Li, J. Wilstrup, R. Jessen, and D. Petrich, "A new method for jitter decomposition through its distribution tail fitting," in Proceedings International Test Conference, 1999, pp. 788-794.

[4] Q. Dou and J. A. Abraham, "Jitter decomposition by time lag correlation," in 7th Inter. Symp. on Quality Electronic Design (ISQED'06), March 2006, pp. 6 pp.-530.

[5] H. Pang, J. Zhu, and W. Huang, "Jitter decomposition by fast Fourier transform and time lag correlation," in 2009 Inter. Conf. on Communications, Circuits and Systems, July 2009, pp. 365-368.

[6] G. Anthonys, M. J. Cree, and L. Streeter, "Jitter extraction in a noisy signal by fast fourier transform and time lag correlation," Applied Mechanics and Materials, vol. 884, pp. 113-121, 92018.

[7] W. Tuttlebee, Software defined radio: origins, drivers and international perspectives, 1st ed. Wiley, 2002.

[8] J. Mitola, "Software radios: Survey, critical evaluation and future directions," IEEE Aerospace and Electronic Systems Magazine, vol. 8, no. 4, pp. 25-36, 1993.

[9] J. R. Machado-Fernández, "Software defined radio: Basic principles and applications," Facultad de Ingeniería, vol. 24, no. 38, pp. 79-96, 2015.

[10] R. Lackey and D. W. Upmal, "Speakeasy: the military software radio," IEEE Communications Magazine, vol. 33, no. 5, pp. 56-61, 1995.

[11] D. M. Davenport, "Wireless communication with a mobile asset employing dynamic configuration of a software defined radio," Aug. 30 2005, US Patent 6,937,877.

[12] P. S. Hall, P. Gardner, and A. Faraone, "Antenna requirements for software defined and cognitive radios," Proceedings of the IEEE, vol. 100 , no. 7, pp. 2262-2270, 2012.

[13] F. K. Jondral, "Software-defined radio: basics and evolution to cognitive radio," EURASIP journal on wireless communications and networking, vol. 2005, no. 3, pp. 275-283, 2005.

[14] W. H. Tuttlebee, "Software-defined radio: facets of a developing technology," IEEE Personal Communications, vol. 6, no. 2, pp. 38-44, 1999.

[15] G. Anthonys, M. J. Cree, and L. Streeter, "Signal processing approaches for jitter extraction in Time-of-Flight range imaging cameras," in 12th International Conference on Signal Processing and Communication Systems (ICSPCS), Cairns, Australia, Dec. 2018.

[16] H. Rapp, M. Frank, F. Hamprecht, and B. Jhne, "A theoretical and experimental investigation of the systematic errors and statistical uncertainties of time-of-flight-cameras," Int. J. Inte. Sys. Tech. and Appl., vol. 5, no. $3 / 4,2008$.

[17] D. Falie and V. Buzuloiu, "Noise characteristics of 3D time-of-flight cameras," in 2007 International Symposium on Signals, Circuits and Systems, vol. 1, July 2007, pp. 1-4.

[18] S. Orfanidis, Introduction to Signal Processing. Prentice Hall, 1996.

[19] R. B. Blackman and J. W. Tukey, "The measurement of power spectra from the point of view of communications engineering part i," The Bell System Technical Journal, vol. 37, no. 1, pp. 185-282, Jan 1958.

[20] R. G. Lyons, Understanding Digital Signal Processing, 3rd ed. Boston, MA: Pearson, 2011.

[21] R. B. Randall, Frequency Analysis, 3rd ed. Brül \& Kjor, 1987.

[22] ABOUT RTL-SDR. https://www.rtl-sdr.com/about-rtl-sdr/. 